\title{
Notulæ ad Plantas Japoniæ et Coreæ. XII.
}

\author{
auctore
}

Takenoshin Nakai, Rigakuhakushi.

170) Scleria tokusanensis, NAKAI. sp. nov.

Cæspitosa gracilis. Caulis alato-triangularis glaberrimus cum foliis usque $15 \mathrm{~cm}$. altus. Folia linearia $1.5-2 \mathrm{~mm}$. lata, radicalia $1-2$, caulina $1-2$ usque $10 \mathrm{~cm}$. longa basi vaginantia.

Inflorescentia laxe racemoso-umbellata v. recemus solitarius. Bracteæ et perianthia dorso et margine barbata late lanceolata v. lanceolata falcata acuminata. Fructus albi lævi sphærici $1.5 \mathrm{~mm}$. longi reticulati glaberrimi.

Hab. Corea austr.: Toksan (T. Mori n. 34 bis).

Planta endemica!

Clavis specierum Scleriæ Coreanæ.

$1\left\{\begin{array}{cccccccccc}\text { Caulis gracillimus filiformis. } & \text { Glomeruli florum spicatim et distante } \\ \text { dispositi } & \ldots & \ldots & \ldots & \ldots & \ldots & \ldots & \ldots & \ldots & \text { S. pergracilis, KuNTH. } \\ \text { Caulis subalato-trigona } & \text { v. } & \text { trigona. } & \text { Inflorescentia non spicata... } & 2 .\end{array}\right.$

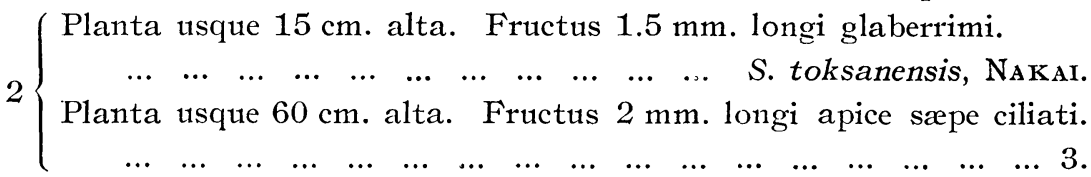

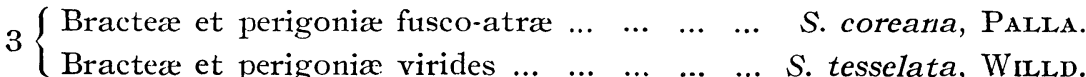

171) Dioscorea nipponica, Makino Illus. Fl. Jap. I. t. 45.

var. pubescens, NakaI.

Folia supra pulverulento-pilosa, infra pubescentia. Cetera ut typica. Hab. Corea: Phyöng-an bor.: Pyök-dang (H. Imai n. 49) Hamgyöng bor.: Manjyokusan (T. NAKAI).

Planta endemica!

173) Salix graciliglans, NAKAI. sp. nov.

Proxima ad S. gracilistyla (S. Thunbergiana) quæ et in Japonia et in Corea vulgatissime crescit, sed differt exqua foliis glabrioribus, spicis 
angustioribus, squamis inflorescentiæ ovatis v. oblongo-ovatis obtusiusculis, ciliis squamis brevioribus vestitis, capsulis minoribus ovato-oblongis.

Caulis prostratus $\mathrm{v}$. ascendens usque $5-6$ pedalis sed sæpe 1-2 pedalis et socialiter supra inundata vestitus, præter apicem glaber flavus sed sæpe rubescens. Folia turionum usque $11 \mathrm{~cm}$. longa $3.2 \mathrm{~cm}$. lata supra glaberrima, infra primo sericea et glauca, demum glabrescentia et glaucina, utrinque acuminata, margine punctato remoteque serrulata, medio latissima.

Flores $\uparrow$ mihi ignoti. Amenta $q$ subcotanea sessilis, maturata 7 mm. lata $3-5 \mathrm{~cm}$. longa densa. Squamæ supra medium atratæ, utrinque ciliis $1 \mathrm{~mm}$. longis hirsutæ $1.5 \mathrm{~mm}$. longæ ovate v. oblongo-ovatæ apice obtusæ v. obtusiusculæ. Carpella dense sericea ovato-oblonga $2.5 \mathrm{~mm}$. longa subsessilia. Styli persistentes cum stigmate indiviso v. bifido $\mathbf{1 . 5}$ $\mathrm{mm}$. longi. Glans angusta fere $1 \mathrm{~mm}$. longa.

Nom. Jap. Chôsen-neko-yanagi.

Hab. Corea: Phyöng-an: Kang-gei (Mrrus n. 312). ad ripas flum. Pôtongkan (H. Imar n. 89) Pyeng-yang (H. Imai n. 55). Hoang-Hai: Hai-jyu (?).

Kyöng-san: Andong (R. K. Smírir n. 27).

Planta endemica!

174) Salix kangensis, NAKAI. sp. nov.

Species proxima ad $S$. daphnoides, sed differt foliis angustioribus et capsulis et stipitibus pilosis.

Ramus cum amenta fæminea tantum mihi notus.

Ramus flavus glaberrimus. Folia initio convoluta subulata, glaberrima, subtus glaucina, breviter petiolata, margine minutissime serrulata acutissima. Amenta of $4-4.5 \mathrm{~cm}$. longa fere $1 \mathrm{~cm}$. lata sessilia cotanea. Squamæ atræe ellipticæ v. ovato-ellipticæ $1.5 \mathrm{~mm}$. longæ $1 \mathrm{~mm}$. latæ longe sericeo-barbatæ, ciliis $2 \mathrm{~mm}$. longis. Glans trapeziformis $0.5 \mathrm{~mm}$. longa. Capsula lanceolata basi subito contracta cum stipite $1 \mathrm{~mm}$. longo parce pilosella. Stylus cum stigmate leviter quadrifido 1.2-1.5 mm. longus glaberrimus.

Hab. Corea: Phyöng-an: Kang-gei (Mrlıs n. 301).

Planta endemica!

175) Corispermum Stauntoni, Moquin Chenop. Enum. p. 104 et in DC. Prodr. XIII. ii. p. 140. Kom. Fl. Mansh. II p. 159.

Hab. Corea: Ham-gyöng: Kan-heung (KIM).

Kyöng-geni: Inchon (Uchryama).

Distr. China et Manshuria. 
176) Menispermum daxuricum, DC. Syst. I. (1818). p. 540.

var. pilosum, Schneid. Illus. Handb. Laubholzk. I. p. 326. fig. 205. a. Diels Menisp. p. 258.

In nostris speciminibus folia omnia late ovata, interdum angulata supra præter venas sparsim pilosas glaberrima subtus toto facie pilosa. Hab. Corea: Ham-gyöng bor.: mons Musanryöng (T. NAKAI). Ham-gyöng austr.: in silvis secus fl. Chang-jyn-gang (T. NAKAI n. 2101).

Distr. ?

\section{Violæ Coreanæ.}

Conspectus Sectionum.

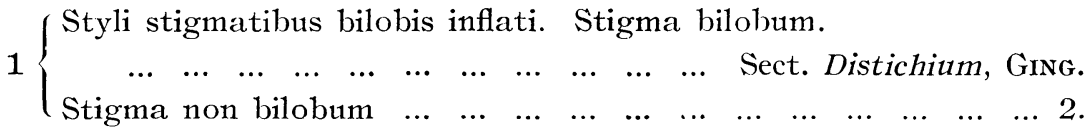

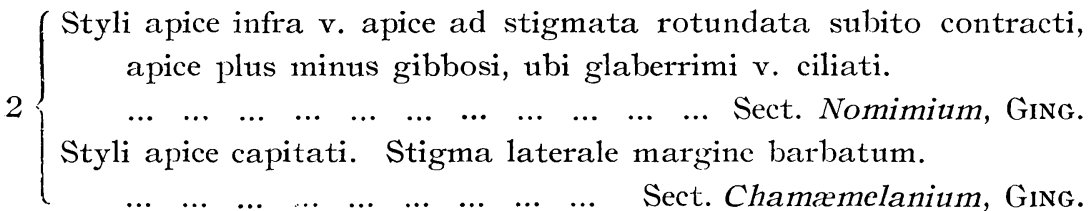

Sect. I. Distichium, Gingrns mss. apud DC. Prodr. I. (1824) p. 300. Leneb. Fl. Ross. I. (1842) p. 254. Maxim. in Mél. Biol. IV. (1876) p' 746 (excl. $V$. verecunda).

(Folia crassiuscula glaberrima. Petala infima usque 11-13 mm. $\begin{array}{llllllllllll}\text { longa. } & \ldots & \ldots & \ldots & \ldots & \ldots & \ldots & \ldots & \ldots & \ldots & \ldots & V . \text { crassa, Makino. }\end{array}$

Folia membranacea adpresse sparsimque pilosa. Petala infima usque

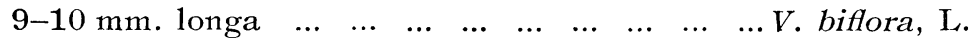

177) (Sp. 1) Viola crassa, Makino in Tokyo Bot. Mag. XIX. p. 87.

V. biflora v. crassifolia, Makrno in Tokyo Bot. Mag. XVI. p. 139.

Hab. Corea sept.: in alpinis montis Waigalbon $2000 \mathrm{~m}$. et supra (T. NAKAI n. 1584).

Distr. Nippon.

178) (Sp. 2) Viola biflora, L. Sp. Pl. p. 936. NakaI F1. Kor. I. p. 66. Hab. Corea sept.: in silvis Picea ajanensis pede montis Waigalbon 1450-1500 m. (T. NAKaI n. 1517). in silvis Picea ajanensis summo montis Shintô-ryöng $1460 \mathrm{~m}$. (T. NAKAI). In via ab oppido Musang meridiem versus Fluvium Tumingan. Vallis Segel-su (Komarov n. 1115).

Distr. Europa, Sibiria, Manshuria, Japonia et America bor. 
Sect. II. Nomimium, Ging. apud DC. 1. c. p. 291. Maxim. 1. c. p. 717. Nominium, Ledeb. Fl. Ross. I. p. 243.

Distichium, Maxım. 1. c. p. 749. p. p.

Conspectus specierum.

$1\left\{\begin{array}{lllllll}\text { Caulis epigeus saltem in fructu elongatus foliaceus } & \ldots & \ldots & \ldots & \ldots & 2 \text {. }\end{array}\right.$

$2\left\{\begin{array}{lllllllllllll}\text { Folia omnia lanceolata } & \ldots & \ldots & \ldots & \ldots & \ldots & \ldots & \ldots & \ldots & \ldots & \ldots & \ldots & 3 . \\ \text { Folia }\end{array}\right.$

Folia saltem partim cordata v. reniformia v. ovata $\quad \ldots \quad \ldots . . .44$.

(Folia utrinque acuminata argute incurvato-serrata. Stipulæ lanceo-

$3\left\{\begin{array}{ccccccccccc}\text { latæe } & \ldots & \ldots & \ldots & \ldots & \ldots & \ldots & \ldots & \ldots & \ldots & V . \text { Websteri, Hemsley. }\end{array}\right.$

Folia basi truncata v. cordata integra v. obscure crenata. Stipula magnæ integræ v. pauci-dentatæ ... ...V. Raddeana, REGEL.

(Folia cordato-reniformia. Stipulæ lanceolatw integræ v. pauciden-

$4\{$ tatæ vulgo magnæ. Flores albi puxpureo-striati. Calcar breve.

$\begin{array}{lllllllll}\text { Folia cordata } \mathrm{v} \text {. ovata } \mathrm{v} \text {. ovato-oblonga } & \ldots & \ldots & \ldots & \ldots & \ldots & \ldots & 5\end{array}$

(Petala alba purpureo-striata, lateralia barbata. Folia cordato-

$5\{$ Stipulæ magnæ lanceolatæ laceratæ... ... v. typica, NAKAI.

$\left\{\begin{array}{l}\text { Stipulæ lineares laceratæ... } \\ \text {... }\end{array}\right.$

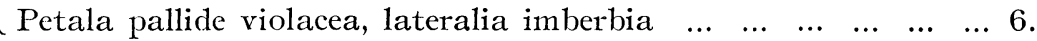

Folia superiora ovato-oblonga v. late lanceolata. Calcar longum.

$6\left\{\begin{array}{ccccccccccc}\ldots & \ldots & \ldots & \ldots & \ldots & \ldots & \ldots & \ldots & \ldots & \ldots & \text { V. ovato-oblonga, MAKino. }\end{array}\right.$

Folia omnia cordato-ovata v. ovata...

(Calcar sepalis brevius. Stipulæ lanceolatæ dentatæ sed haud fim$\begin{array}{lllllllllll}\text { briatæ } & \ldots & \ldots & \ldots & \ldots & \ldots & \ldots & \ldots & \ldots & \ldots & \ldots\end{array}$

7 Calcar sepalis longius. Stipulæ lineares fimbriato-laceratæ.

$\begin{array}{llllllllllll}\ldots & \ldots & \ldots & \ldots & \ldots & \ldots & \ldots & \ldots & \ldots & \ldots & \ldots & V . \text { grypoceras, A. Gray. }\end{array}$ variant petala alba $\ldots . . . . \quad \ldots . .$. forma albiflora, Makino.

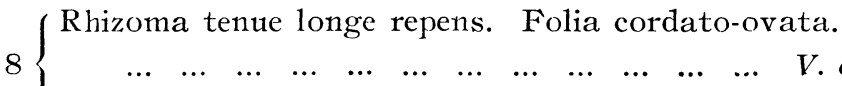

$\left\{\begin{array}{rrrrrrrrrrrrr}\ldots & \ldots & \ldots & \ldots & \ldots & \ldots & \ldots & \ldots & \ldots & \ldots & \ldots & \ldots & V \text {. epipsila, Leder. } \\ \text { Rhizoma }\end{array}\right.$

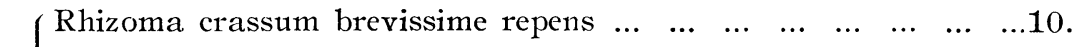
$9\left\{\begin{array}{c}\text { Rhizoma columnale } v \text {. interdum innovatio cum internodio elongato } \\ \text { a annotino distans }\end{array} \ldots\right.$

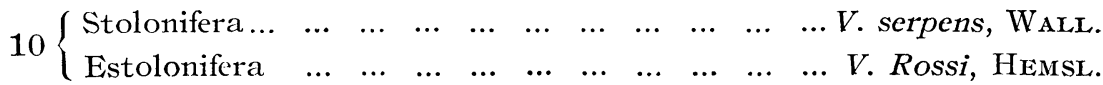

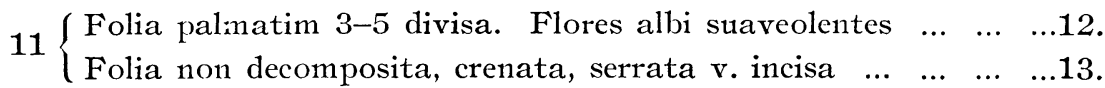


12

$\left\{\begin{array}{l}\text { Lacini foliorum lanceolati . } \\ \text { Lacini foliorum lineares }\end{array}\right.$

V. dissecta, LEDEB.

v. chrophylloides, Makino.

$\left\{\begin{array}{llllllllllllll}\text { Petioli vix alati } & \ldots & \ldots & \ldots & \ldots & \ldots & \ldots & \ldots & \ldots & \ldots & \ldots & \ldots & \ldots & \ldots\end{array}\right.$

(Flores albi. Petala lateralia fauce barbata. Folia et pedunculi vulgo glaberrima rarius minute ciliolata... ... V. Patrini, DC.

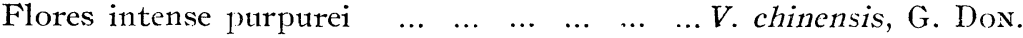

$14\left\{\begin{array}{l}\text { Petioli et pedunculi glaberrimi } \ldots \\ \text { Petioli et }\end{array}\right.$

b $\left\{\begin{array}{l}\text { Petala lateralia imberbia } \ldots \\ \text {... }\end{array}\right.$ Petala lateralia fauce barbata ... ... $\beta$. transtica, NAKAI.

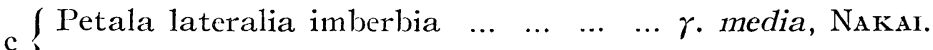
I Petala lateralia fauce barbata ... $\ldots . . . \quad$.. ciliata, NAKAI.

$15\left\{\begin{array}{lllllllllll}\text { Folia ovato-oblonga v. ovata } & \ldots & \ldots & \ldots & \ldots & \ldots & \ldots & \ldots & \ldots & \ldots 16 \\ \text { Folia cordata }\end{array}\right.$

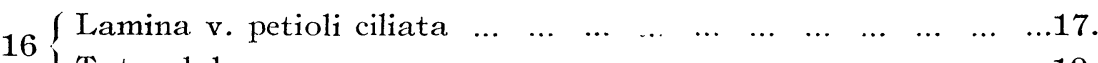
( \begin{tabular}{lllllllllllllll} 
Tota glabra & $\ldots$ & $\ldots$ & $\ldots$ & $\ldots$ & $\ldots$ & $\ldots$ & $\ldots$ & $\ldots$ & $\ldots$ & $\ldots$ & $\ldots$ & $\ldots$ & $\ldots$ & $\ldots 19$ \\
\hline
\end{tabular}

(Petioli et pedicelli albo-hirsuti v. griseo-tomentosi. Lamina glabra v. ciliolata lanceolato-oblonga. Petala claro-purpurea.

$\begin{array}{llllllllllll}\ldots & \ldots & \ldots & \ldots & \ldots & \ldots & \ldots & \ldots & \ldots & \ldots & \ldots & V \text {. hirtipes, S. Moore. }\end{array}$

(Petioli et pedicelli hirtelli. Lamina supra glabra. \begin{tabular}{llllllllllll}
$\ldots$ & $\ldots$ & $\ldots$ & $\ldots$ & $\ldots$ & $\ldots$ & $\ldots$ & $\ldots$ & $\ldots$ & $\ldots$ & $\ldots$ & $\%$ \\
\hline typica, NaKaI.
\end{tabular} Petioli et pedicelli griseo-tomentosi. Lamina supra plus

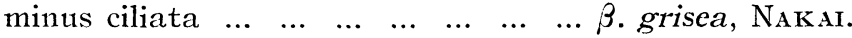

Petioli et pedicelli glabri v. adpresse ciliati non hirsuti... $\quad . . \quad \ldots 18$.

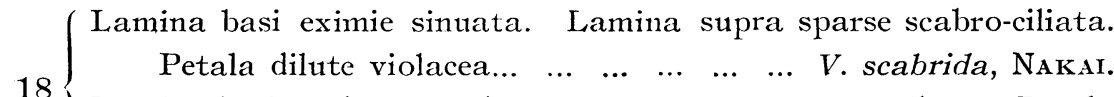

18 Lamina basi cordata v. subtruncata. Lamina supra pilosa. Petala $\begin{array}{llllllll}\text { claro-purpurea ... } & \ldots & \ldots & \ldots & \ldots & \ldots & V . & \text { phalacrocarpa, Maxin. }\end{array}$

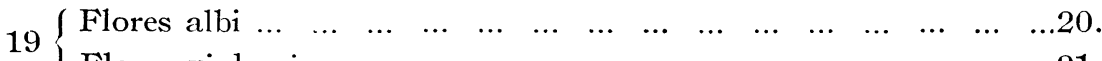

$\left\{\begin{array}{llllllllllllll}\text { Flores violacei } \ldots & \ldots & \ldots & \ldots & \ldots & \ldots & \ldots & \ldots & \ldots & \ldots & \ldots & \ldots & \ldots & \ldots 21 .\end{array}\right.$

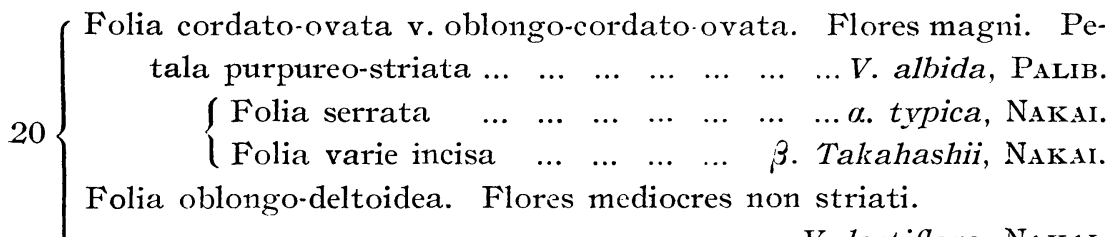
$\begin{array}{lllllllllllll}\ldots & \ldots & \ldots & \ldots & \ldots & \ldots & \ldots & \ldots & \ldots & \ldots & \ldots & \ldots & \text { V. lactiflora, NAKAI. }\end{array}$ 


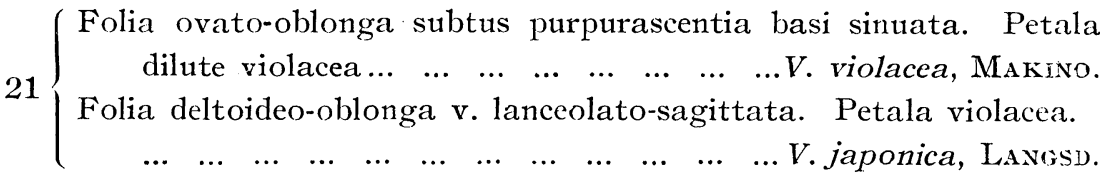

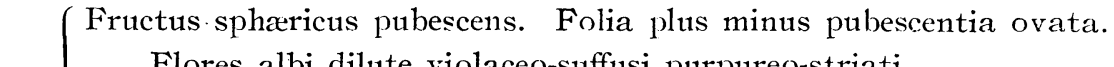
22 $\begin{array}{lllllllllll}\ldots & \ldots & \ldots & \ldots & \ldots & \ldots & \ldots & \ldots & \ldots & V \text {. hirta, L. v. collina, REGEL. }\end{array}$ $\begin{array}{lllllllll}\text { Fructus oblongus v. ellipticus glaber ... } & \ldots & \ldots & \ldots & \ldots & \ldots & \ldots & \ldots 23\end{array}$ $23\left\{\begin{array}{llllllllllllllll}\text { Flores albi } & \ldots & \ldots & \ldots & \ldots & \ldots & \ldots & \ldots & \ldots & \ldots & \ldots & \ldots & \ldots & \ldots & \ldots & \ldots 24 .\end{array}\right.$ ( Flores dilute violacei v. purpurei v. intense purpurei $\quad \ldots \quad \ldots \quad \ldots 25$.

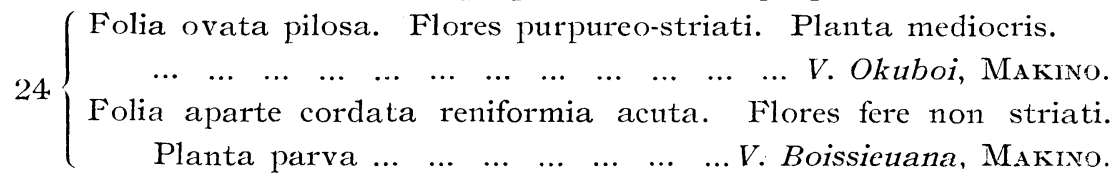

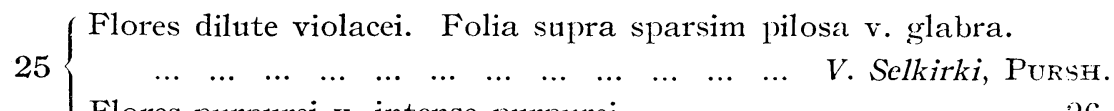

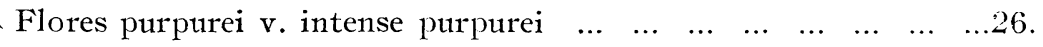

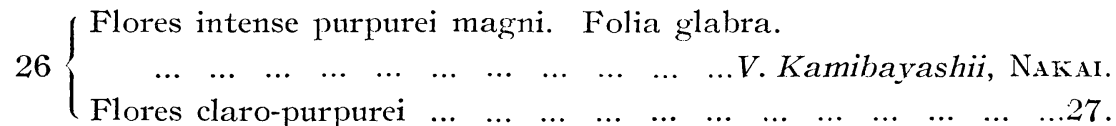

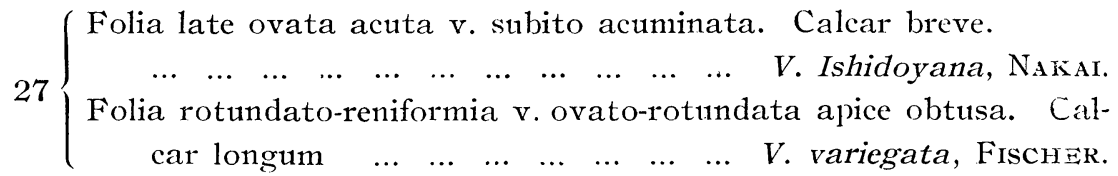
Folia infra purpurascentia supra variegata. $\left\{\begin{array}{ccccccccccc}\ldots & \ldots & \ldots & \ldots & \ldots & \ldots & \ldots & \ldots & \ldots & \ldots & \ldots \\ \text { Folia } & \ldots & \ldots & \ldots & \text { typica, Regeria viridia } & \ldots & \ldots & & & \end{array}\right.$

179) (Sp. 3) Viola Websteri, Hemsley in Journ. Linn. Soc. XXIII. p.

56. NakaI F1. Kor. I. p. 64.

Hab. Corea sept.: in trajectu Laoling (WEBsTER).

Planta endemica!

180) (Sp. 4) Viola Raddeana, Regel Pl. Radd. I. p.p. 251. 256. t 7. f. 1-5. NakaI F1. Kor. I. p. 64. II. p. 445.

Hab. Corea media: in turfosis montium Kan-uonto (FAURIE $n$. 304.) in herbidis Ouensan (T. NakaI).

Corea sept.: mons Paik-pyök-san (T. Ishidoy a n. 44).

Distr. Nippon, Manshuria et Amur.

181) (Sp. 5) Viola verecunda, A. Gray Bot. Jap. p. 382. NakaI Fl. Kor. I. p. 65. II. p. 445.

Nom. Vern. Him-maruma-jappul (Quelpært). 
Hab. Quelpært.: in herbidis Hongno (TAQUeT n. 4583. 4576. 2643) sub muris agrorum Hongno (TAQUET $n .4596$ ) in agris Hongno (TAqueT n. 4792) in sepibus Hongno (TaqueT n. 5398. 4588 ) in herbidis (TAQUET n. 2637) secus torrentes Hiotien (TAqUeT n. 4580) in herbidis pede montis Hallasan (T. Nakar). Corea media: Yang-jyn (T. NakaI) Seoul (T. Mori n. 29) Suigen (H. Ueki n. 71) Namsan (T. Mori et T. Uchiyama) Paukhan (Sontag) Ouensan (T. NakaI) Pyeng-yang (H. ImaI) mons Kum-gang-san (T. Uсhiуama).

Corea sept.: Kang-gei (Milss n. 386).

Distr. China, Formosa, Manshuria et Japonia.

182 ) (Sp. 6) Viola acuminata, Ledeb. Fl. Ross. I. p. 252. NaKaI

Chôsenshokubutsu I. (1914) p. 120. Fl. Kor. II. (1911) p. 445.

V. canina, L. var. acuminata, REgeL Pl. Radd. I. p. 247. NAKAI Fl. Kor. I. p. 65.

Differt a $V$. canina caule elatiore, foliis majoribus et acuminatis, stipulis 2-5 plo majoribus, calcaribus duplo brevioribus, petalis albis.

«. typica, NakaI.

Stipulæ foliacex laciniatæ.

Hab. Corea austr.: mons Chirisan 1300 m. (T. NAKaI n. 720). Corea sept.: Kang-gei (MrLus n. 382).

Distr. Sibiria, Dahuria, Baical, Manshuria, China bor., Yeso et Nippon.

var. intermedia, NAKAI.

Stipulæ profunde laciniatæ, lacinis angustissimis.

Hab. Quelpært: in silvis Hioten (TAQUeT n. 2650) in herbidis Peptyangi (Taguet n. 2636).

Corea austr.: Andong (R. K. Smith) Chôjyo (T. NakaI) Mons Pai-yang-san ('T. NAKaI n. 1105).

Corea media: Koumpho insulæ Pôtô (Y. Hanabusa) mons Namsan (T. Uchiyama) mons Namhansan ( $T$. Uchiyama) mons Peukhansan (T. Uchiyama) mons Kasan (H. Ueki n. 135) mons Taiseisan (ImaI).

Corea sept.: Seisin (T. NakaI) Musan-ryöng (T. NAKaI) Nyagsan (Ishidoy A n. 43).

Distr. Manshuria et Nippon.

183) (Sp. 7) Viola grypoceras, A. Gray in Perry's Exped. II. p. 308. Makino in Tokyo Bot. Mag. XXVI (1912) p. 152.

V. sylvestris, Kıт. v. grypoceras, Maxım. in Mél. Biol. IX. p. 743.

$V$. coreana, DE BoIss. 
V. longe-pedunculata, Fr. et SAv. Enum. Pl. Jap. II. p. 286.

V. silvestris v. japonica, (GIng.) Makino in Tokyo Bot. Mag. XVI. p. 146.

V. canina var.? japonica, Ging. in DC. Prodr. I. (1824) p. 298.

Differt a $V$. canina, calcaribus elongatis, petalis lateralibus imberbibus.

Hab. Quelpært: in sepibus Hongno (TAQueT n. 182. 4977 T. Mori n. 71) mons Hallasan ('T. Nakai n. 880).

Corea austr.: insula Wangtô (T. NAKAI).

Distr. Nippon, Shikoku et Kiusiu.

forma albiflora, Makino in Tokyo Bot. Mag. XXVI. p. 152.

Hab. Quelpart: in silvis $600 \mathrm{~m}$. (TAQUET n. 4581) secus torrentes Hiotien (TAQUeT n. 3518) Hoatien (TaQUeT n. 2646) in silvis $500 \mathrm{~m}$. (Taquet 11.6047 ) in sepibus Hongno (TAQUET n. 4593, 4577, 4585) in silvis supra Hioton (TAQUET n. 4584).

Distr. Nippon.

184) (Sp. 8) Viola koraiensis, Nakar. sp. nov.

Species affinis $V$. silvestris et ejus var. sabulosa, V. Reichenbachiana, $V$. riviana etc., sed ex omnibus petalis lateralibus imberbibus, calcare breve differt. Viola grypoceras etiam proxime venit, sed calcaribus longioribus, stipulis fimbriatis ex hac ipse statim dignoscenda.

Radix perennis, foliis emortuis atris squamosis dense obtecta. Caulis glaberrimus. Stipulæ lanceolatæ integræ v. serratæ v. fere fimbriatoserrata virides. Folia longe petiolata cordato-rotundata obtusa v. acuta crenulato-serrata glaberrima. Flores axillares solitarii. Pedunculi glabri graciles folia superantes. Sepala lineari-lanceolata v. linearia viridia. Petala pallide violacea late oblanceolata obtusa imberbia. Calcar breve 2-2.5 $\mathrm{mm}$. longum. Capsula elliptica acuta $7-8 \mathrm{~mm}$. longa.

Hab. Corea sept.: in silvis Picea ajanensis montis Sôdenrei 1580 m. (T. NAKAI n. 1502) in silvis Larix Principis-Ruprechtii pede montis Paiktusan $1500-2000 \mathrm{~m}$. (T. Mori n. 75. T. NAKAI) in pumiceis montis Paiktusan 2100-2400 m. (T. $\mathrm{NA}_{\mathrm{A}}$ KaI et T. Mori).

Planta endemica!

185) (Sp. 9) Viola ovato-oblcnga, (Mig.) Makino in Tokyo Bot. Mag. XXI (1907) p. 57. NakaI Report Veg. Isl. Quelpært (1914) p. 65. n. 913.

V. silvestris forma ovato-oblonga, MiQ. Prol. Fl. Jap. p. 86.

V. silvestris var. ovato-oblonga, Makino in Tokyo Bot. Mag. XVI (1902) p. 137. 
V. silvestris v. montana, Yatabe in Tokyo Bot. Mag. VI (1892) p. 131.

Hab. Quelpært: mons Hallasan $500 \mathrm{~m}$. (T. NAKAI).

Distr. Nippon.

186) (Sp. 10) Viola serpens, W (1824) p. 296. Nakai Fl. Kor. I. p. 68.

Hab. Corca media: in silvis montis Kum-gang-san (T. UCHIYAMA).

Distr. Malaya, India, China et Manshuria.

187) (Sp. 11) Viola Rossi, HemsL. in Journ. Linn. Soc. XXIII. p. 54. Nakar Fl. Kor. I. p. 66. II. p. 446.

Hab. Quelpært: in silvis supra Hioton (TAQUET n. 4578).

Corea austr.: in herbidis Fusan (FAURIE n. 614) in silvis insulae Wangtô (T. NAKaI) in silvis montium Chirisan (T. NaKaI n. $438)$.

Corea media: in silvis Koang-nyong (T. NaKaI) mons Hichihôsan (Ueki n. 56) mons Peuk-han-san (T. Mori) mons Namsan (T. Uchiyama).

Corea scpt.: Pyok-dang (H. Imai).

Distr. China, Manshuria et Nippon.

18 ) (Sp. 12) Viola epipsila, Leder. Fl. Ross. I. p. 247. NaKai Fl. Kor. I. p. 67.

Hab. Corea sept.: Fluvium Tumingan. In via ab oppido Musang meridiem versus vallis Segel-su (V. Komarov n. 1107) in silvis Picea ajanensis montis Sodenrei 1500 m. (T. NakaI n. 1650). Distr. Sibiria, Amur et Manshuria.

189 ) (Sp. 13) Viola dissecta, Ledeb. Fl. Alt. I. (1829) p. 255.

var. chærophylloides, (Regel) Makino in Tokyo Bot. Mag. XXVI (1912) p. 153.

V. pinnata $\delta$ chærophylloides, REGEL Pl. Radd. I. (1861) 1. 232.

$V$. dissecta v. cherophylloides subvar. typica, Makino 1. c.

V. pinnata, (non L.) Nakai F1. Kor. I. p. 68. II. p. 445.

Hab. Quelprert: in dumosis Hongno (TAQUET n. 4999) in silvis (Faurie, Mori, Taquet n. 38).

Corea austr.: in silvis Pomasa (Faurie n. 617).

Corea media: mons Namsan (T. Uсhiуama) mons Penkhansan (T. Uchiyama).

Corea sept.: Sensan (H. IMaI) Seikoshin (T. NakaI).

Distr. Amur, Manshuria, Kiusiu et Shikoku.

var. Sieboldiana, (MAXıм.) NAKAI Chôsenshokubutsu I. (1914) p. 124. 
$V$. pinnata var. Sieboldiana, Maxım. in Mél. Biol. IX. (1876) p. 718. Fr. et Sav. Enum. Pl. Jap. II. p. 646.

V. chærophylloides v. Sieboldiana, Makino in Tokyo Bot. Mag. XIX. 1. 87 .

V. Sieboldiana, Makino in Tokyo Bot. Mag. XIX. p. 144.

$V$. dissecta v. chærophylloides subvar. a. typica forma Sieboldiana, Makino in Tokyo Bot. Mag. XXVI. p. 154.

Hab. Quelpart: mons Hallasan (T. NakaI).

Corea austr.: mons Chirisan (T. NAK.II 11. 402) Urusan (N. OKADA).

Corea media: mons Hichihôsan (H. Lekr n. 57) mons Penkhansan (T. Mori) Seoul (Sontag).

Corca sept.: Kang-gei (Mrrls n. 376).

Distr.: Manshuria, Kiusiu, Shikoku et Nippon.

190) (Sp. 14) Viola Patrini, UC. mss. apud DC. Prodr. I. p. 293. NakaI Fl. Kor. I. p. 71. II. 446.

Hab. Quelpart: in agris (Faurie, Taquet n. 2634) Hoatien (TAQUeT n. 2645) in silvis $600 \mathrm{~m}$. (TAQUeT n. 4582, 4814) circa Tshe-jyu (T. NakaI).

Corea austr.: Riri (T. NAKAI) Chô-jỹo (T. NAKAI) Andong (Sмiтh n. 15).

Corea media: Koang-nyong (T. NAKaI) Giseifu (T. NakaI) Suigen (Rishôko) Pyeng-yang (H. Imai).

Corea sept.: Punyong (T. Nakar).

Distr. India, China, Manshuria et Japonia.

191) (Sp. 15) Viola chinensis, G. Don Gard. Syst. I. (1831) p. 322. Nakai Fl. Kor. II. p. 446.

V. Patrini var. chinensis, DC. Prodr. I. p. 293. NakaI Fl. Kor. I. p. 71 .

a. typica, Nakar.

Glabra. Petala lateralia imberbia.

Hab. Corea media: Inchon (T. Uchiyama).

Corca austr.: Mok-pho (T. Uсhiуama).

ß. transitica, NAKAI.

Glabra. Petala lateralia fauce barbata.

Hab. Quelpært: mons Hallasan (T. NaKar n. 988).

Corea austr.: Noryong (T. NakaI) Andong (R. K. Smith n. 16).

Corea media: Koang-nyong (T. NAKAI) Yang-jyu (T. NAKaI) Chang-ho-uön (T. Uchiyama) Seoul (Sontag).

Corea sept.: Anjyu (Mills n. 373). 


\section{$\gamma$. media, NAKAI.}

Petioli et pedicelli puberuli. Petala imberbia.

Hab. Corea media: inter Chemulpo et Seoul (Sontag) Ouensan (T. NakaI) Suigen (H. UEKI).

o. ciliata, NAKAI.

Petioli et pedicelli puberuli. Petala fauce barbata.

Hab. Quelpært: circa Tshedju (T. NAKaI).

Corea austr.: Riri (T. NakaI).

Corea media: mons Perkhansan (T. Mori) Namsandong ('T. UChiy ama).

Distr. species. China, Manshuria et Japonia.

Specimina sequentia petiolos et pedicellos pilosos portant, sed flores mihi ignoti.

Hab. Corea austr.: Kurei (T. NAKAI).

Corea media: Namsan (T. Uchiyama).

Sequentia sunt glabra.

Hab. Corea media: mons Namhansan, mons Namsan, mons Paukhansan ( $T$. Uchiyama).

Specimina posteriore colloco a Faurie et TAquet in Corea et in Quelpært sunt lecta. Observavi ca olim in suis herbariis, sed petalis cilias eorum non visis, nunc in varietatibus non distinguere possum.

Hab. Quelpart: in agris Hongno (TAQUET n. 179. 4586. 4594. 4791). in pratis Hallasan $1500 \mathrm{~m}$. (Faurie n. 1748) in herbidis Hokemi (Taquet n. 2642) circa Hongno (TaqueT n. 80 ) in herbidis (TaqueT n. 2632) in herbidis Syckeni (TaQUeT n. 2649) Hoatien (TAQUET n. 2647).

Corea media: in herbiclis Seoul (TaQueT n. 2629).

191) (Sp. 16) Viola hirtipes, S. Moore in Journ. Linn. Soc. XVII. p. 379. Nakai Fl. Kor. I. p. 70. II. 1. 446.

\%. typica, NAKAI.

Folia supra glabra. Petioli et pedicellihirsuti.

Hab. Quelpart: in herbidis $1000 \mathrm{~m}$. (Taqurer n. 4119).

Corea austr.: Chojyo (T. NAKAI).

Corea media: Koang nyong (T. Nakar, T. Mori n. 319) Seoul (Son'TAG).

Corea sept.: Kang-gei (Mrr.Ls 11. 385).

Distr. China bor., Manshuria et Japonia.

3. grisea, NakaI in Tokyo Bot. Mag. XXVII (1913) p. 129. nom. nud.

Petioli et pedicelli griseo-tomentosi. Lamina supra pilosa. 
Hab. Quelprert: mons Hallasan $1800 \mathrm{~m}$. (T. NAKaI),

Corea media: mons Peukhansan (T. Mori).

Planta endemica!

192) (Sp. 17) Viola scabrida, NakaI in Matsum. Icon Pl. Koish. II. t. 128 (1914) excl. stigma male delineatum. et in Tokyo Bot. Mag. XXVIII (1914) p. 312.

V. Selkirki, (non Pursh) NAKAl Fl. Kor. II. p. 447. p.p. et Report Veg. M't. Chirisan p. 40 n. 333.

Hab. Corea media: Koang-nyong (T. NAKAI).

Corea sept.: mons Musanryöng (T. NAKAI).

Corea austr.: mons Chirisan $1000 \mathrm{~m}$. (T. NAKAI) in silvis insulæe Wangtô $600 \mathrm{~m}$. (T. NAKAI).

Planta endemica!

193) (Sp. 18) Viola phalacrocarpa, Maxım. in Mél. Biol. IX. (1876)

p. 726. NakaI F1. Kor. I. p. 70. JI. p. 446. Report Veg. Isl. Quelpært p. 66. n. 915. Report Veg. M't. Chirisan p. 40 m. 331.

Hab. Quelpært: mons Hallasan (T. NaKaI n. 928) Ikiri (T. NakaI) Hongno (T. NAKaI) secus vias Hongno (TaQUeT $n$. 4590 ) in herbidis $900 \mathrm{~m}$. ('TAQueT n. 4998) in sepibus Hongno (TAQUET n. 4793) in campis Hallasan $600 \mathrm{~m}$. (FAURIE n. 1749). Corea media: in herbidis Seoul (Taquet n. 2631) Seoul (SonTAG) Koang-nyong (T. NakaI) mons Namsan (T. Uchiyama) Inchon ( $T$. Uchiyama) mons Peukhansan (T. Uchiyama) Seoul (Ishidoya) Yang-jyu (T. NakaI) Ouensan (T. NAKaI) insula Pôtô (Y. Hanabusa).

Corea austr.: Mokpo (T. UchiyanA).

Corea sept.: Anjyu (Mrlus n. 374) Kang-gei (Mrlıs n. 384).

Distr. Manshuria et Japonia.

194) (Sp. 19) Viola albida, Palib. Consp. Fl. Kor. I. p. 31. t. II. f. 2. NakaI Fl. Kor. I. p. 68. II. p. 446. Report. Veg. Isl. Wangtô p. 11. Report Veg. M't. Chirisan p. 40 n. 328.

o. typica, NakaI.

$V$. dissecta v. chærophylloides, subvar. albida, Makino in Tokyo Bot. Mag. XXVI (1912) p. 155.

V. dissecta v. alhida, NakaI in Matsun. Icon. P1. Koish. I. pl. 47 (1912).

Hab. Corea media: mons Peukhansan (T. Uchiyama) Seoul (Sontag) mons Namsan (Ishidoya n. 42. T. Mori) Koangnyong (T. NAKAI). 
Corea austr.: mons Chirisan (T. NAKAI 616. Mori 232) Wangtô (T. NAKAI).

ß. Takahashii, (Makino) Nakai.

V. dissecta v. chærophylloides subvar. multifida, Makino in Tokyo Bot. Mag. XXVI (1912) p. 154 (excl. syn. V. pinnata v. multifida REGEL).

V. dissecta v. chærophylloides subvar. Takahashii, Makino mss. apud Makino 1. c.

Hab. Corea media: Koang-nyong (T. NAKAI) mons Namsan (T. Mori).

Corea austr.: insula Wangtô (T. NAKaI 11. 606).

Planta endemica!

In specimina exsiccata hæc species cum var. Takahashii in Viola dissecta sensim transire videtur, sed foliis ambitu ovatis v. late ovatis, floribus inodoratis exqua statim dignoscenda.

195) (Sp. 20) Viola lactiflora, NakaI in Tokyo Bot. Mag. XXVIII (1914) p. 329.

Hab. Corea austr.: in herbidis inter Seiyu et Chôjyo (' $\mathrm{T}$. NakaI n. 1044).

Distr. China.

196) (Sp. 21) Viola violacea, Makino Illus. Fl. Jaj. I. p. 17 t. 67. NAKaI Report Veg. Isl. Quelpært p. 66. n. 920.

Hab. Quelpært: in silvis montis Hallasan $800 \mathrm{~m}$. (TAQUeT 1 . 565 ) in silvis $600 \mathrm{~m}$. (T. NakaI).

Distr. Kiusiu, Shikoku et Nippon.

197) (Sp. 22) Viola japonica, LangSD. ex Fischer in litt. apud DC. Prodr. I. p. 294. NakaI Fl. Kor. I. p. 73. II. p. 447. Report Veg. Isl. Quelpært p. 65. n. 911.

Hab. Quelpart: in herbidis littoris (TAQUE' n. 4992) in agris Hongno (Taquet n. 4591, 4597, 4575) in herbidis Hongno (TAQUET n. 2648) in herbidis Hiotien (TAQUE' n. 2627) in sepibus Hongno (Taquet n. 5397) in herbidis $500 \mathrm{~m}$. (TaqueT n. 4598).

Corea media: in herbidis Seoul (Faurie n. 613) Chang-nyongri, mons Namsan, mons Namhansan et mons Peukhansan (T. Uchiyama) Seoul (Sontag).

Distr. Japonia, China et India.

198) (Sp. 23) Viola hirta, L. Sp. P1. p. 934.

var. collina, Regel P1. Radd. I. p. 236. NaKAI Fl. Kor. I. p. 67. V. Selkirki, Kom. Fl. Mansh. III. p. 59. p.p. 
Hab. Corea media: mons Paiyangsan (T. NAKAI n. 1027).

Corea media: Ouensan (T. NAkaI) Koang-nyong ( T. Nakar et T. Mori n. 170) mons Kum-gang-san (T. Uchiyama) Namsan ( $T$. Uchiуama).

Corea sept.: Ho-jyo (H. ImaI) Kôzanchin (H. ImaI) Hekidô (H. ImaI) Fluvium Jalu superior. Vallis Tadinpen (V. Komarov n. 1108).

Distr. Sarmatia, Sibiria, Amur et Manshuria.

Viola hondoensis ( $=V$. hirta v. japonica, Maxim.) huic proxime venit et est cum Viola Keiskei, MrQ. quæ a botanicis Japonicis olim cum Vjola Okuboi commixta identica.

199) (Sp. 24). Yiola Okuboi, Makino in Tokyo Bot. Mag. XVII. p. 85.

๙. typica, Makino 1. c.

Hab. Quelpært: in sylvis 600 m. (TAQueT n. 4579) in silvis Peptjyang (TAqueT n. 2640) in silvis $400 \mathrm{~m}$. (TAqueT n. 6052) in herbidis littoris (TAQUET n. 4592).

Corea austr. insula Wangtô (T. NAKaI n. 832) Via inter Süiyu et Chôjyô (T. NAKar n. 1206).

Distr. Japonia.

200) (Sp. 25) Viola Boissieuana, Makino Tokyo Bot. Mag. XVII. (1902) p. 127. Nakai Rep. Veg. Isl. Quelpært. p. 65. n. 906.

Hab. Quelpart: in silvis Abies nephrolepis montis Hallasan $1800 \mathrm{~m}$. ('T. NAKAI).

Distr. Nippon.

201) ( $\left.\mathrm{S}_{\mathrm{p}} .26\right)$ Viola Selkirki, Pursh mss. apud Goldre in Edinb. Phil. Journ. (1822) p. 319. NAKaI Fl. Kor. I. p. 73.

Hab. Quelpært: secus vias Mokan (Taquet n. 4589) in petrosis silvarum (FAURIE n. 1752) in agris Hongno (TAQUET n. 1s1) Yengsil $1000 \mathrm{~m}$. (TaqueT n. 4121) in silvis Abies nephrolepis montis Hallasan $1500 \mathrm{~m}$. (T. NAKAI 11. 1291).

Corea austr. in silvis et in petrosis montium Chirisan (T. NAKAI 11. 636).

Corea sept. in silvis Laricis pede montis Paiktusan $1480 \mathrm{~m}$. (T. NAKAI).

Distr. Sarmatia, Suecia Norvegia, Sibiria, Dahuria, Kamtschatica, Manshuria, Amur, Nippon, Sachalin et Canada.

202) (Sp. 27) Viola Kamibayashii, NakaI. sp. nov.

$V$. albida, NakAI Fl. Kor. I. p. 68. p.p.

Paulum simulans ad Viola hirtipes si ejus cilii omittentur. 
Rhizoma repens breve crassum exquo radices albos emittit. Folia longe petiolata glaberrima. Stipulæ integræ preter apicem petiolo aduatæ 5-10 mm. longæ. Petioli usque $18 \mathrm{~cm}$. longi sulcati sed apice paulum alati v. fere exalati. Lamina cordata v. late ovata basi sinu aparto, apice acuta margine incurvato remote crenulata e sinu ad apicem usque $8 \mathrm{~cm}$. longa et $7 \mathrm{~cm}$. lata, subtus pallidiora. Pedunculi usquc 10 $\mathrm{cm}$. longi crassi glaberrimi, bracteis binis infra medium positis. Sepala viridia lanceolata attenuata $8-10 \mathrm{~mm}$. longa, appendice postica quadrangulare $2 \mathrm{~mm}$. longa. Petala violacea $v$. pallide violacea, fauce pallida et intensius striata, suprema reflexa usque $20 \mathrm{~mm}$. longa late oblanceolata apice emarginata. Calcar 4-5 $\mathrm{mm}$. longum 2-4 mm. latum. Capsula fere $1-1.5 \mathrm{~cm}$. longa obtuse trigona ellpsoidea. Semina fuscoflava.

Nom. Jap. Kô-ryô-sumirc.

Hab. Corea: Chöl-là: insula Ok-tô (T. NakaI n. 810).

Kyöng-geui: Koang-nyong ( $T$. Ishidoya).

Planta endemica!

203) (Sp. 28) Viola Ishidoyana, NakaI in Tokyo Bot. Mag. XXVII (1913) p. 129 nom. nud.

V. Selkirki, (non Pursh.) Kom. Fl. Mansh. III. p. 59. p.p.

Acaulis. Radix columnalis, sed primo tenuis. Folia omnia radicalia glaberrima. Stipulæ angustæ lineares usque medio arlnatr. Lamina ovata v. late ovata, acuta v. acuminata, crenata infra pallidiora, hasi aparte cordata, glabra v. secus venas ciliolata. Pedicelli glaberrimi foliis breviores, versus apice ciliolati. Calcar $5 \mathrm{~mm}$. longum $3 \mathrm{~mm}$. latum. Petala claro-purpurea. Fructus ellipsoidei 5-7 mm. longi.

Hab. Corea sept.: mons Pyak-pyök-san (Ismidora) Pyök-dang (H. Imai n. 246).

Corea media: Hasonri (T. Uchiyama).

Corea austr.: Toksan ( $T$. Mori).

Distr. Manshuria.

204) (Sp. 29) Viola variegata, Fischer in litt. apud DC'. Prodr. I. (1824) 1. 293.

v. typica, Rfgel Pl. Radd. I. (1861) p. 226.

Hab. Corea media: Koang-nyong (T. NakaI) mons Peukhansan (T. Mori et T. Uchiyama) mons Namsan (T. Uchiyama). Corea austr.: mons Chirisan (T. MoRI n. 231. T. NAKAI) mons Paiyangsan (T. NAKaI n. 1131).

Corca sept.: Matinryöng (A. Mishina) Sosan (H. Imai) mons Musan-ryöng (T. NAKaI). 
Distr. China bor., Japonia, Sachalin, Manshuria, Ussuri et Dahuria.

var. ircutiana, (Turcz.) Regel 1. c. Kom. Fl. Mansh. III. p. 59.

V. ircutiana, Turcz. Fl. Baic. Dah. I. p. 180. Leder. Fl. Ross. I. p. 244 .

Hab. Corea sept.: Kang-gei (MrLls n. 375) ad ripas fl. Tumingang (K. Jô).

Distr. Sibiria, Amur et Manshuria.

Sect. III. Chamæmelanium, Gingrns mss. apud DC. Prodr. I. 1). 300. Ledeb. Fl. Ross. I. p. 254. Maxim. in Mél. Biol. IX. p. 751. Tauber'T in Pflanzenf. III. vi. p. 335.

205) (Sp. 30) Viola uniflora, L. Sp. Pl. p. 936. NAKaI Fl. Kor. 1. p. 64. II. p. 445, Report Veg. Is1. Quclpart p. 66. n. 918, Report Veg. Isl. Wangtô p. 11.

Hab. Quelpart: in silvis Hallasan (TAQUET 11. 2628. 4120). in petrosis siccis montis Hallasan $2000 \mathrm{~m}$. (Faurie n. 1750) in monte Hallasan $1800 \mathrm{~m}$. (T. NAKAI 11. 1287) in monte Hatlasan $1900 \mathrm{~m}$. ('T. NAKAI) in silvis montis Hallasan $500 \mathrm{~m}$. (T. NAKaI).

Corea austr.: Ulsan (N. OKADA) in silvis insulæe Wangtô 400 m. (T. NAKAI).

Corea media: mons Peukhansan (T. Uchiyama) Ouensan ( $T$. NakAI) Seoul (Son'tag).

Corea sept.: mons Matin-ryöng (A. Mishima) mons Paik-pyöksan (T. Ishidoya) Musang (K. Marda) Kang-gei (Mrlls u. $378)$.

Distr. Sibiria, China, Manshuria, Kiusiu et Nippon occid.

Rhododendron Sect. Viscidula, Matsum. et Nakai.

Gemma florifera terminales. Flores brevissime racemosi penduli.

Corolla campanulata, lobis 5 conformibus. Stamina 10 .

Ovarium glanduloso-pilosum.

Species unica.

206) Rhododendron nipponicum, Matsum. in Tokyo Bot. Mag. XIII. (1899) p. 17. Komatsu in Matsum. Icon. Pl. Koish. Vol. I. (1911) t. 5 .

Hab. Nippon: circa montis Shimizutôge (T. Suzuxi) mons Adzumasan (G. NAKAHARA) pede montis Tateyama (YATABE et Matsumura) mons Gassan (Yatabe et Matsumura).

Planta endemica! 
207) Ajuga (Genevenses) spectabilis, NAKaI sp. nov.

Primo obtutu $A$. incisæ similis esse videtur, sed folia basi cuneata.

Rhizoma repens et stolones emittit. Caulis usque $50 \mathrm{~cm}$. altus glaberrimus robustus. Folia opposita, radicalia mihi ignota, caulina inferiora emarcida parva, superiora late elliptica apice acuminata argute grosse irregulariterque serrata et ciliolata, supra sparsissime ciliolata, infra pallidiora et secus venas sparsissime pilosa, basi ad petiolem brevem attenuata usque $17 \mathrm{~cm}$. longa $9 \mathrm{~cm}$. lata. Spica solitaria terminalis densa interdum foliacea $3 \mathrm{~cm}$. longa. Pedicelli glaberrimi v. parcissime ciliolati. Cupula obovata parce pilosa 10 -nervis $2.5 \mathrm{~mm}$. longa, lacinis linearibus trinerviis $5 \mathrm{~mm}$. longis. Corolla violaceo-carulea, tubo 15 $\mathrm{mm}$. longo ad apicem sensim inflato, labio superiore obtuso, margine albo-ciliato $1.5 \mathrm{~mm}$. longo $3 \mathrm{~mm}$. lato, labio inferiore 3 -fido, labio medio maximo late obovato leviter emarginato. Stamina libera hirtella exerta. Anthera atra. Styli leviter exerti glaberrimi apice bifido, limbo postico longiore. Semen ignotum.

Hab. Corea: Kyöng-geni: Koang-nyong ( $\mathrm{T}$. Mor et Y. AkABANE).

Planta endemica!

\section{8) Sambucus latipinna, NaKar.}

Ramus fructifer tantum mihi notus.

Ramus glaberrimus exsiccatus nigricans. Folia bijugo imparipinnata glaberrima. Foliola terminalia petiolulis $2.7 \mathrm{~cm}$. longis, fere rotundata apice subito attenuata, preter actmine $9.5 \mathrm{~cm}$. longa $8.5 \mathrm{~cm}$. lata basi acuta, lateralia breviter petiolulata elliptica et subito acuminata cum acumine $9.5 \mathrm{~cm}$. longa $6 \mathrm{~cm}$. lata basi subtruncata, omnia arguta serrata. Inflorescentia cymoso-paniculata apice plana glaberrima. Fructus $3 \mathrm{~mm}$. longi maturi rubri.

Hab. Corea: Hoang-Hai: Chang-tang.

Planta endemica!

209) Ainsliæa acerifolia, Schelz in ZorLING. Cat. p. 126.

var. subapoda, NAKAI.

Rhizoma repens. Caulis terminalis brevis v. subnullus. Folia late ovata grosse serrata sed indivisa.

Hab. Corea: Phying-an bor.: Nyag-sui-dong (T. Ishidoya).

Planta endemica! 